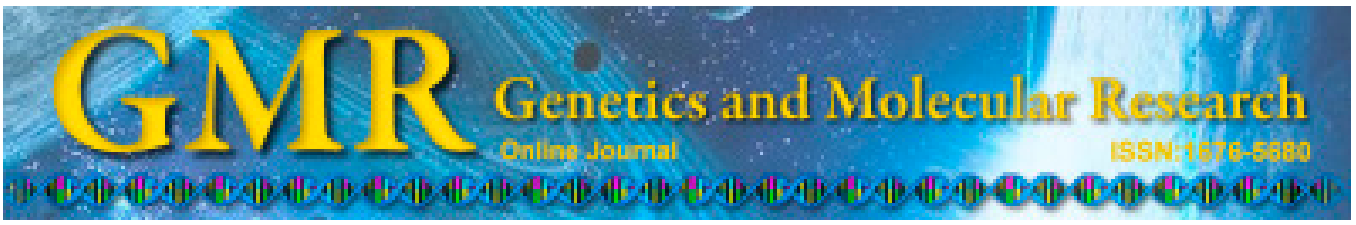

\title{
Protective effect and mechanism of probucol in the treatment of spinal cord injury
}

\author{
W.B. Zhu' ${ }^{1}$ Y.H. Wang ${ }^{1}$, G.F. Sun $^{2}$ and J.H. Wu ${ }^{3}$ \\ ${ }^{1}$ Department of Anesthesiology, Binzhou People's Hospital, \\ Shangdong, China \\ ${ }^{2}$ Department of Orthopedics and Traumatology, Binzhou People's Hospital, \\ Shangdong, China \\ ${ }^{3}$ Department of Otolaryngology, Binzhou People's Hospital, \\ Shangdong, China \\ Corresponding author: W.B. Zhu \\ E-mail: WeiboZhu2068@163.com
}

Genet. Mol. Res. 14 (3): 8029-8037 (2015)

Received January 26, 2015

Accepted April 6, 2015

Published July 17, 2015

DOI http://dx.doi.org/10.4238/2015.July.17.11

\begin{abstract}
To investigate the effects of probucol on the treatment of spinal cord injury in rat, 80 rats were randomly divided into two groups of 40: a group treated with probucol and a control group. Allen's method was used to establish a rat model of spinal cord injury. After establishment, probucol $\left(500 \mathrm{mg} \cdot \mathrm{kg}^{-1} \cdot \mathrm{day}^{-1}\right)$ was intraperitoneally injected into the treatment group rats for 1 week, while the same amount of saline was used to treat the control group. On days 1, 7, 14, 21, and 28 after treatment, the function of rats' spinal cord was evaluated according to the Bresnahan locomotor rating scale. Serum protein and mRNA levels of the cytokines [interferon (IFN)- $\gamma$, tumor necrosis factor (TNF)- $\alpha$, and interleukin (IL)-17] were measured using enzyme-linked immunosorbent assay and quantitative polymerase chain reaction, respectively. Protein levels of IFN- $\gamma$, TNF- $\alpha$, IL-17, and the downstream markers signal transducer and activator of transcription (STAT)-1 and STAT-3 were measured using western blot. In addition, the oxidative stress-related parameters, superoxide dismutase (SOD) and malondialdehyde (MDA), were also measured. It was found that
\end{abstract}


compared to control group, rats from the treatment group had significantly lower levels of IFN- $\gamma$, TNF- $\alpha$, and IL-17 $(\mathrm{P}<0.05)$ on days 1 and 7 , as well as lower MDA levels and higher SOD activity on days 7, 21, and $28(\mathrm{P}<$ 0.05). In summary, probucol improved the recovery of locomotion function after spinal cord injury in rats through downregulation of inflammation and upregulation of anti-oxidative activity.

Key words: Probucol; Spinal Cord Injury; Oxidative stress; Inflammation

\section{INTRODUCTION}

In recent years, an increase in the incidence of accidents on roads, construction sites, and in mines has resulted in spinal cord injuries (SCI) becoming a common type of traumatic injury seen in orthopedic and neurology departments (Song et al., 2013). Compared to other types of traumatic injury, SCI has a poor prognosis and a higher chance of causing disability, which seriously affects a patient's quality of life (Reglodi et al., 2012; Wanner et al., 2013; Xianbao et al., 2013). Previous studies have demonstrated the important role of inflammation and oxidative stress in the pathogenesis of SCI. Therefore, preventing secondary or progressive injury to spinal cord nerve cells is a key therapeutic approach to improve prognosis (Reglodi et al., 2012). In this study, we used a rat model of SCI to investigate the mechanism of action of probucol, an anti-oxidative stress drug that has recently been shown to be clinically effective.

\section{MATERIAL AND METHODS}

\section{Materials}

Rats were used for all experiments, and all procedures were approved by the Animal Ethics Committee of Binzhou People's Hospital (Shandong, China). Female Wistar rats (aged 6 to 8-weeks-old, 150-200 g) were used to establish an SCI model. All rats were housed in a specific-pathogen-free (SPF) environment, with a temperature of 23 to $27^{\circ} \mathrm{C}$, and humidity of 60 to $70 \%$. Enzyme-linked immunosorbent assay (ELISA) kits for the measurement of interferon (IFN)- $\gamma$, tumor necrosis factor (TNF)- $\alpha$, and interleukin (IL)-17 were purchased from USCN Life Science Inc. (Wuhan, China). Monoclonal antibodies for western blot analysis of the expressions of IFN- $\gamma$, TNF- $\alpha$, IL-17, and signal transducer and activator of transcription (STAT)-1 and STAT-3 were purchased from Cell Signaling Technology (MA, USA). Superoxide dismutase (SOD) activity and malondialdehyde (MDA) levels were measured using ELISA kits (Sangon, Shanghai, China).

\section{Establishment of SCI rat model and treatment}

An SCI rat model was established using Allen's method (Bao et al., 2011). Briefly, rats were anesthetized with an intraperitoneal injection of $2 \%$ sodium pentobarbital (100 mg/ $\mathrm{kg})$, and back hair was removed using sodium sulfide. Following an incision $(\sim 3 \mathrm{~cm})$ centered on T10, a dorsal laminectomy was performed from $\mathrm{T} 9$ to $\mathrm{T} 11$, and the spinous process was removed to expose the spinal dura mater. The exposed spinal cord was injured using an impact rod with a weight of $30 \mathrm{~g}$ and diameter of $3 \mathrm{~mm}$, striking from a height of $10 \mathrm{~cm}$ with damage 
energy of $300 \mathrm{~J}$. Control rats underwent the same process without the impact rod. The incisions were sutured, and green enzyme was intraperitoneally injected for 3 days $(40000 \mathrm{U} / \mathrm{d})$ (Liu et al., 2011). The criteria for a successful establishment of SCI included appearance of tail swing reflex, retraction flutter, and paralysis of two hind limbs.

To treat the induced SCI, probucol was intraperitoneally injected into the rats of the treatment group ( $\left.500 \mathrm{mg} \cdot \mathrm{kg}^{-1} \cdot \mathrm{day}^{-1}\right)$. The same dose of saline was administered to the control group. In case of uroschesis or cystorrhexis following induction of SCI, bladder massage was performed to assist uresis 2 to 3 times daily.

\section{Evaluation of locomotion function}

The classic Basso, Beattie, and Bresnahan locomotor rating scale (BBB) was used to evaluate the locomotor function of SCI rats. The locomotor function of normal rats and completely paralyzed rats were defined as scores of 21 and 0 , respectively - a higher score corresponded to a better locomotion function (Reglodi et al., 2012). The score was assessed using a $100-\mathrm{cm}$ disk with one rat at a time. Each rat was monitored for 4 min once per day. The monitoring time was at 8 p.m. when the rats were usually more active.

\section{Assessment of oxidative stress}

SOD activity and MDA levels were measured in the spinal cord to assess oxidative stress. Higher SOD activity indicates stronger anti-oxidative effect, and a higher MDA level indicates an increase in oxidative activity (Esposito et al., 2012). Rats were sacrificed by intraperitoneally injecting an overdose of sodium pentobarbital, and subsequently spinal cord tissues $(20 \mathrm{mg})$ were extracted and placed in a $1.5-\mathrm{mL}$ Eppendorf tube. A small amount of saline was added to the tube to keep the extracted tissues moist. Cell homogenate was obtained using an ultrasonic cell crusher (McEwen et al., 2011). SOD activity and MDA level were measured using a commercial kit (Sangon, Shanghai) following manufacturer protocols.

\section{Measurement of inflammation}

ELISA was used to detect the level of inflammatory cytokines in serum. Reverse transcriptase polymerase chain reaction (RT-PCR) and western blotting were used to measure the mRNA and protein levels of IFN- $\gamma$, TNF- $\alpha$, and IL-17, respectively. Western blotting was also used to measure the expression of STAT-1 and STAT- 3 and the extent of phosphorylation. The detailed methods were as follows.

On days $0,3,7,14,21$, and 28 after treatment with probucol, blood ( 3 to $5 \mathrm{~mL}$ ) was drawn from the retro-orbital of rats and left for $2 \mathrm{~h}$ at $4^{\circ} \mathrm{C}$. This was followed by low speed centrifugation to obtain the supernatant, which was stored at $-80^{\circ} \mathrm{C}$ (Maggio et al., 2012). The ELISA assay was performed according to manufacturer protocol once all the samples had been collected. Repeated freezing and thawing was avoided.

RT-PCR was used to measure mRNA levels at the indicated time points. In rats that showed significant differences in mRNA expression, western blotting was used to measure protein expression on the seventh day after treatment. 


\section{RT-PCR}

Total RNA was extracted from fresh tissues using Trizole (Takara) according to the manufacturer protocol. Subsequently, RNA was reverse transcribed to cDNA using a kit (Takara) followed by real time quantitative-PCR (RT-qPCR). The conditions of RT-qPCR were as follows (Schonberg et al., 2012): 30 cycles of $94^{\circ} \mathrm{C}$ for $30 \mathrm{~s} ; 57^{\circ} \mathrm{C}$ for $30 \mathrm{~s}$ (annealing), $72^{\circ} \mathrm{C}$ for $30 \mathrm{~s}, 72^{\circ} \mathrm{C}$ for $1 \mathrm{~min}$ (final extension). The primers are shown in Table 1.

\begin{tabular}{ll}
\multicolumn{1}{c}{ Table 1. RT-PCR primers. } \\
\hline Gene & Primers \\
\hline IFN- $\gamma$ & Forward: 5'-ATGAGTGCTACACGCCGCGTCTTGG-3' \\
& Reverse: 5'-GAG TTCATTGACAGCTTTGTGCTGG-3' \\
TNF- $\alpha$ & Forward: 5'-CCACGTCGTAGCAGCAAACCACCAAG-3' \\
& Reverse: 5'-CAGGTACATGGGCTCCTCATA-CC-3' \\
IL-17 & Forward: 5'-GAAGAGGGAGCCTGAGAAGT-3' \\
& Reverse: 5'-TAAAGCCACAGAAAAACAG-3' \\
$\beta$-actin & Forward: 5'-ACCTCCAACACCCCAGCCATG-3' \\
& Reverse: 5'-CTGATCACATCTGCTGGAAGGTGG-3' \\
\hline
\end{tabular}

\section{Western blot}

Spinal cord tissues were lysed with lysis buffer and incubated in ice. Following centrifugation, the supernatant was isolated. After measuring concentration using the bicinchoninic acid (BCA) assay, the supernatant was incubated in a $100^{\circ} \mathrm{C}$ water bath for $5 \mathrm{~min}$ and was run using $10 \%$ sodium dodecyl sulfate polyacrylamide gel electrophoresis (SDS-PAGE). After that, it was transferred to polyvinylidene difluoride (PVDF) membranes for $2 \mathrm{~h}$, blocked for $1 \mathrm{~h}$ with $5 \%$ nonfat milk, and then washed thrice with Tris-buffered saline and Tween 20 (TBS-T). After washing, primary antibodies (1/1000 dilution) against IFN- $\gamma$, TNF- $\alpha$, IL17, STAT-1, and STAT-3 (Cell Signal Technology) were added and incubated overnight. The membranes were again washed thrice with TBS-T, secondary antibody (1/1000 dilution) was added, and the samples were incubated for $1 \mathrm{~h}$. Proteins were visualized using a chemiluminescence system (Rabchevsky et al., 2011).

\section{Statistical analysis}

The SPSS version 17.0 software was used to analyze the data. Categorical data were analyzed by chi-square test, and measurement data were analyzed by the $t$-test or mean variance. Data are reported as means $\pm \mathrm{SD} . \mathrm{P}<0.05$ was considered to be statistically significant.

\section{RESULTS}

\section{Function recovery of rats post-surgery}

Locomotion scores in rats from the treatment group were significantly higher than those in the control group $(\mathrm{P}<0.05)$ on days $7,14,21$, and 28 following surgery. The differences between the treatment group and the control increased over time (Table 2). 


Table 2. Locomotion scores of rats in different group (means \pm SD).
\begin{tabular}{lccccc}
\hline Group & \multicolumn{5}{c}{ Locomotion scores post-surgery } \\
\cline { 2 - 6 } & Day 1 & Day 7 & Day 14 & Day 21 & Day 28 \\
\hline Treatment & $0.62 \pm 0.21$ & $6.78 \pm 1.21$ & $9.23 \pm 2.19$ & $12.14 \pm 3.29$ & $18.82 \pm 6.22$ \\
Control & $0.66 \pm 0.19$ & $3.29 \pm 1.32$ & $5.77 \pm 2.28$ & $6.22 \pm 1.98$ & $7.49 \pm 2.12$ \\
F & 1.023 & 5.264 & 4.283 & 39.298 & 43.19 \\
P & 0.558 & 0.023 & 0.039 & 0.000 & 0.000 \\
\hline
\end{tabular}

\section{Measurement of inflammatory cytokines in serum}

ELISA was used to measure the serum level of IFN- $\gamma$, TNF- $\alpha$, and IL-17. As seen in Table 3, the levels of IFN- $\gamma$, TNF- $\alpha$, and IL-17 peaked from day 1 to day 7 following surgery and then dropped to the baseline on day 14. Cytokine levels were significantly lower in treated rats than in the control group $(\mathrm{P}<0.05)$.

\begin{tabular}{|c|c|c|c|c|c|c|}
\hline Cytokines & & Day 1 & Day 7 & Day 14 & Day 21 & Day 28 \\
\hline \multirow[t]{3}{*}{ IL-17 } & Control & $112.33 \pm 21.22$ & $47.12 \pm 9.21$ & $12.52 \pm 2.19$ & $6.18 \pm 1.77$ & $2.16 \pm 0.32$ \\
\hline & Treatment & $79.89 \pm 12.42$ & $22.59 \pm 7.12$ & $9.32 \pm 3.12$ & $3.18 \pm 0.22$ & $2.20 \pm 0.18$ \\
\hline & $\mathrm{F}, \mathrm{P}$ & $31.232,0.000$ & $9.123,0.001$ & $2.863,0.079$ & $2.112,0.099$ & $1.721,0.882$ \\
\hline \multirow[t]{3}{*}{ IFN- $\gamma$} & Control & $152.33 \pm 31.22$ & $77.12 \pm 29.21$ & $22.52 \pm 6.19$ & $16.18 \pm 6.77$ & $6.16 \pm 1.32$ \\
\hline & Treatment & $89.89 \pm 22.42$ & $32.59 \pm 12.12$ & $16.32 \pm 3.12$ & $12.18 \pm 1.22$ & $4.20 \pm 0.18$ \\
\hline & $\mathrm{F}, \mathrm{P}$ & $30.298,0.000$ & $6.798,0.004$ & $2.192,0.098$ & $1.722,0.781$ & $0.967,0.992$ \\
\hline \multirow{3}{*}{ TNF- $\alpha$} & Control & $92.33 \pm 21.22$ & $67.12 \pm 19.21$ & $32.52 \pm 11.19$ & $5.18 \pm 2.77$ & $1.16 \pm 0.32$ \\
\hline & Treatment & $39.89 \pm 12.42$ & $12.59 \pm 2.12$ & $4.32 \pm 1.12$ & $1.18 \pm 0.22$ & $1.20 \pm 0.18$ \\
\hline & $\mathrm{F}, \mathrm{P}$ & $18.921,0.000$ & $16.229,0.000$ & $9.182,0.000$ & $1.988,0.965$ & $0.432,0.997$ \\
\hline
\end{tabular}

\section{Inflammation infiltration in spinal cord tissues}

RT-qPCR and western blot were used to detect the mRNA and protein expression of IFN- $\gamma$, TNF- $\alpha$, and IL-17 in spinal cord tissues. As seen in Figures 1 and 2, the mRNA and protein expressions of IFN- $\gamma$, TNF- $\alpha$, and IL- 17 were detected in control rats from days 1 to 14 following surgery and reduced over time. Compared to the control at the same time point, rats receiving treatment displayed significantly lower levels of IFN- $\gamma$, TNF- $\alpha$, and IL-17 (P < 0.05) (Figure 1D-F).

Western blot analysis showed that the protein expression of inflammatory cytokines reduced on day 7 following surgery in rats from the treatment group; this was consistent with results of RT-qPCR analysis. In addition, the expression and phosphorylation levels of the downstream inflammation markers STAT-1 and STAT-3 also reduced (Figure 2A and B), suggesting that probucol inhibited both the infiltration of inflammatory cytokines into spinal cord tissues and the activation of downstream signaling.

\section{Analysis of oxidative stress of spinal cord}

Oxidative stress was evaluated by measuring SOD activity and MDA level. An increase in SOD activity indicates that a treatment has had an anti-oxidative effect, and MDA level relates to the concentration of superoxide. As shown in Figure 3A and B, SOD activity 
was significantly higher, and MDA level was significantly lower in treated rats than in control rats $(\mathrm{P}<0.05)($ Table 4$)$.

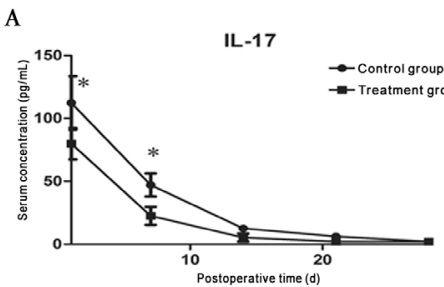

C

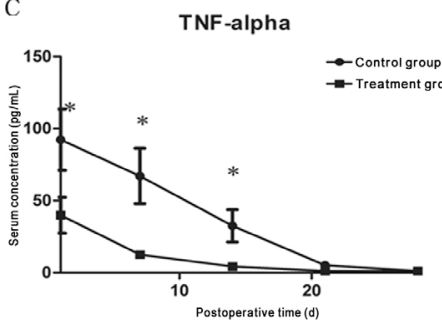

E

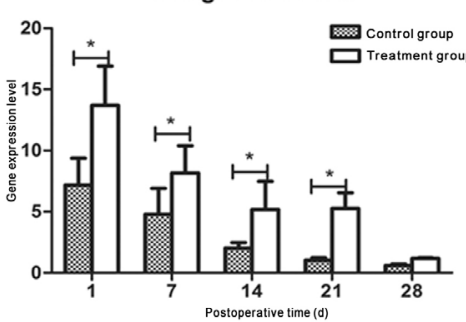

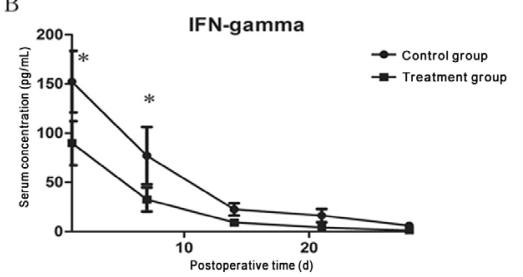

D

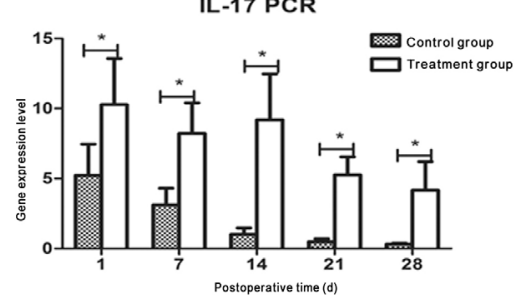

F
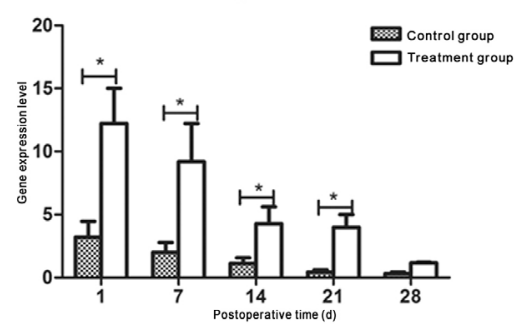

Figure 1. Inflammation evaluation. A.-C. Serum level of interleukin (IL)-17, interferon (IFN)- $\gamma$, and tumor necrosis factor $(\mathrm{TNF})-\alpha(* \mathrm{P}<0.05)$. D.-F. mRNA expression of IL-17, IFN- $\gamma$, and TNF- $\alpha$ in spinal cord tissues $(* \mathrm{P}<0.05)$.

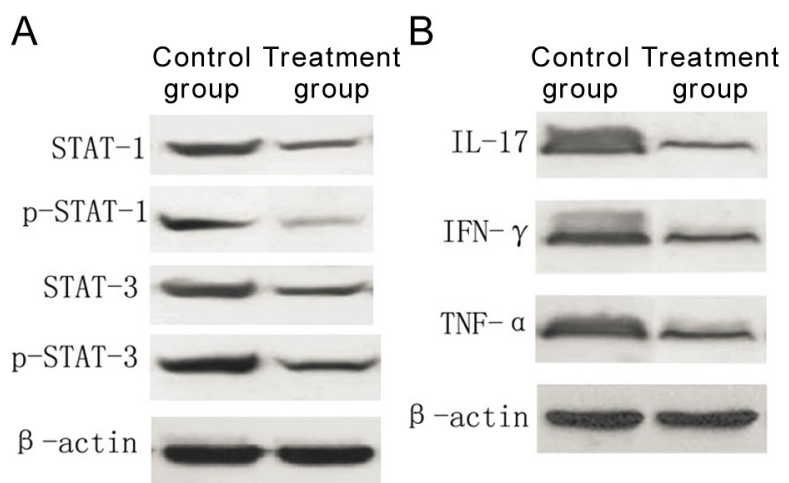

Figure 2. Western blot analysis of inflammation in spinal cord on day 7 following surgery. A. Activation of signal transducer and activator of transcription 1 (STAT-1) and STAT-3. B. Inflammatory cytokines infiltration in spinal cord. 
A

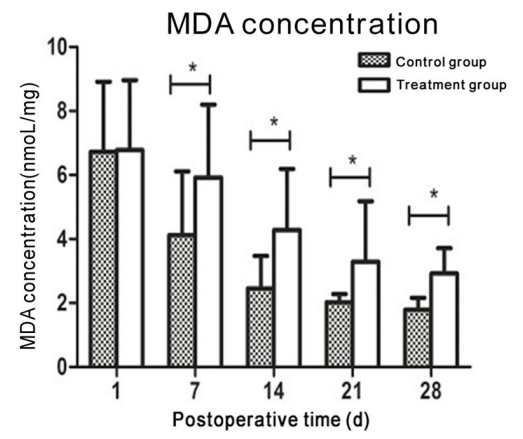

B

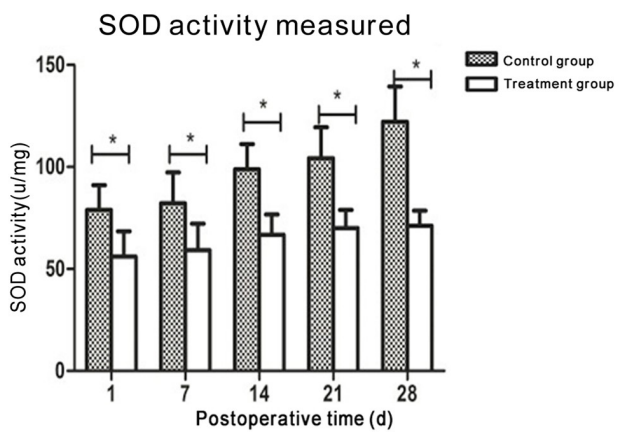

Figure 3. Analysis of oxidative stress in spinal cord of rats. A. Malondialdehyde (MDA) level in spinal cord. B. Superoxide dismutase (SOD) activity in spinal cord. $* \mathrm{P}<0.05$.

Table 4. Oxidative stress analysis of spinal cord tissue samples (means $\pm \mathrm{SD}$ ).

\begin{tabular}{llccccc}
\hline Parameters & & Day 1 & Day 7 & Day 14 & Day 21 & Day 28 \\
\hline MDA (mmol/mg) & Control & $6.78 \pm 2.18$ & $5.92 \pm 2.28$ & $4.28 \pm 1.91$ & $3.29 \pm 1.89$ & $2.92 \pm 0.79$ \\
& Treatment & $6.72 \pm 2.19$ & $4.12 \pm 1.99$ & $2.45 \pm 1.02$ & $2.02 \pm 0.26$ & $1.79 \pm 0.37$ \\
& F, P & $1.721,0.882$ & $2.142,0.047$ & $2.699,0.033$ & $2.155,0.043$ & $2.156,0.043$ \\
SOD (U/mg) & Control & $56.12 \pm 12.28$ & $59.21 \pm 13.02$ & $66.79 \pm 9.91$ & $70.02 \pm 8.89$ & $71.22 \pm 7.29$ \\
& Treatment & $78.95 \pm 12.12$ & $82.16 \pm 15.15$ & $98.79 \pm 12.39$ & $104.29 \pm 15.16$ & $122.19 \pm 17.22$ \\
& F, P & $5.329,0.005$ & $6.149,0.004$ & $7.612,0.001$ & $10.982,0.000$ & $11.182,0.000$ \\
\hline
\end{tabular}

\section{DISCUSSION}

Probucol is an FDA-approved antioxidant used in the clinic and has been reported to be used as an antioxidant and anti-inflammatory agent, and to regulate levels of blood lipid (Bao et al., 2011; Reglodi et al., 2012; Wanner et al., 2013). Probucol is effective in the protection of vascular smooth muscle cells, improving blood vessel function, regulating local lipid metabolism, and preventing restenosis following vascular intervention and/or coronary artery bypass (Liu et al., 2011). In addition, previous studies have shown that probucol could protect neurocytes, cardiomyocytes, and pancreatic $\beta$-cells through downregulation of apoptosis under inflammation and hypoxia (Zeng et al., 2011). It has been postulated that the cytoprotective effect of probucol in the above-mentioned organs is due to its antioxidant and anti-inflammatory properties (Esposito et al., 2012; Ni et al., 2014).

Following injury to the spinal cord, progressive and secondary tissue injury can develop because of inflammation and injury repair. This self-destructive cascade effect could be the main reason for the poor prognosis in patients with spinal cord injury (Myers et al., 2012). In recent years, some studies have demonstrated that after spinal cord injury, local tissue injury and inflammatory cell infiltration leads to inflammation and inflammatory cytokine secretion (Myers et al., 2014). On the other hand, inflammation and hypoxia elevate the concentration of superoxide in injured spinal cord tissues and lead to damage to neurocytes. All of this contributes to spinal cord damage and its associated complications (Hassler et al., 2014); therefore, inflammation and superoxide can be considered the main factors for spinal cord injury. 
Even though probucol has been used in the clinic for many years, there have been no reports of probucol being used to treat spinal cord injuries (Bretzner et al., 2011). Considering the known anti-inflammatory and antioxidant effect of probucol (Inagaki et al., 2012), we sought to evaluate its effect on an animal model of spinal cord injury.

We have successfully established a rat model of spinal cord injury using Allen's method. After inducing spinal cord injury, the locomotion function of lower extremities significantly reduced in rats, and did not improve after 4 weeks. However, after probucol treatment, the locomotion function significantly improved; this suggests that probucol improved the paralysis of the lower extremity in rats.

In order to investigate the mechanism of probucol, we collected blood and spinal cord samples from rats at different time points to measure inflammation. On the seventh day following surgery, we observed both obvious inflammatory cytokine infiltration in serum and spinal cord tissues as well as activation of STAT-1 and STAT-3 in spinal cord, which were consistent with previous studies. On the seventh day following surgery, inflammation was observed in the injured spinal cord and whole body of rats, and this was significantly inhibited after probucol treatment; this indicates that probucol exerted an anti-inflammatory effect in this model. IL-17, IFN- $\gamma$, and TNF- $\alpha$ have been shown to exert apoptotic effects through the STAT-1 and STAT-3 signaling pathways (Massberg et al., 2011), and the inhibition of the above cytokines and pathways resulted in reduced apoptosis which had a protective effect on neurocytes in the spinal cord.

SOD, an enzyme catalyzing the disproportionation of superoxide, has an effect on tissue protection. In the event of tissue injury, SOD is consumed leading to reduced activity (Liu et al., 2011; Myers et al., 2012, 2014; Ni et al., 2014). Increased production of superoxide, also leads to an increase in the level of the metabolic product, MDA. Therefore, MDA elevation can be used as an indicator of peroxidation (Endo et al., 2013). In this study, we demonstrated reduced SOD activity and increased MDA levels after spinal cord injury, which were inhibited after probucol treatment. Under peroxidation, cell damage from oxygen free radicals increased, leading to loss of cell membrane integrity and subsequent susceptibility to damage. Once all the peroxidation-related changes had been inhibited, local cell damage was ameliorated.

In conclusion, inflammation and peroxidation are the main factors that cause spinal cord damage after injury. Probucol reduces inflammation and promotes anti-oxidant activity, and therefore could effectively reduce inflammatory cytokine secretion, inhibit the activation of downstream signaling pathway, improve the extent of local peroxidation, and promote the recovery of locomotion function in rats after spinal cord injury. Although an effective treatment of spinal cord injury in rat models, probucol has not been used in the clinic and therefore requires further investigation.

\section{Conflicts of interest}

The authors declare no conflict of interest.

\section{ACKNOWLEDGMENTS}

We thank the anonymous reviewers for reviewing this manuscript. 


\section{REFERENCES}

Bao F, Brown A, Dekaban GA, Omana V, et al. (2011). CD11d integrin blockade reduces the systemic inflammatory response syndrome after spinal cord injury. Exp. Neurol. 231: 272-283.

Bretzner F, Gilbert F, Baylis F and Brownstone RM (2011). Target populations for first-in-human embryonic stem cell research in spinal cord injury. Cell Stem Cell 8: 468-475.

Endo K, Saiki A, Yamaguchi T, Sakuma K, et al. (2013). Probucol suppresses initiation of chronic hemodialysis therapy and renal dysfunction-related death in diabetic nephropathy patients: Sakura study. J. Atheroscler. Thromb. 20: 494502.

Esposito E, Bruscoli S, Mazzon E, Paterniti I, et al. (2012). Glucocorticoid-induced leucine zipper (GILZ) over-expression in T lymphocytes inhibits inflammation and tissue damage in spinal cord injury. Neurotherapeutics 9: 210-225.

Hassler SN, Johnson KM and Hulsebosch CE (2014). Reactive oxygen species and lipid peroxidation inhibitors reduce mechanical sensitivity in a chronic neuropathic pain model of spinal cord injury in rats. J Neurochem. 131: 413-417.

Inagaki M, Nakagawa-Toyama Y, Nishida M, Nakatani K, et al. (2012). Effect of probucol on antioxidant properties of HDL in patients with heterozygous familial hypercholesterolemia. J. Atheroscler. Thromb. 19: 643-656.

Liu C, Shi Z, Fan L, Zhang C, et al. (2011). Resveratrol improves neuron protection and functional recovery in rat model of spinal cord injury. Brain Res. 1374: 100-109.

Maggio DM, Chatzipanteli K, Masters N, Patel SP, et al. (2012). Acute molecular perturbation of inducible nitric oxide synthase with an antisense approach enhances neuronal preservation and functional recovery after contusive spinal cord injury. J. Neurotrauma 29: 2244-2249.

Massberg S, Byrne RA, Kastrati A, Schulz S, et al. (2011). Polymer-free sirolimus- and probucol-eluting versus new generation zotarolimus-eluting stents in coronary artery disease: the Intracoronary Stenting and Angiographic Results: Test Efficacy of Sirolimus- and Probucol-Eluting versus Zotarolimus-eluting Stents (ISAR-TEST 5) trial. Circulation 124: 624-632.

McEwen ML, Sullivan PG, Rabchevsky AG and Springer JE (2011). Targeting mitochondrial function for the treatment of acute spinal cord injury. Neurotherapeutics 8: 168-179.

Myers SA, DeVries WH, Gruenthal MJ, Andres KR, et al. (2012). Sildenafil improves epicenter vascular perfusion but not hindlimb functional recovery after contusive spinal cord injury in mice. J. Neurotrauma 29: 528-538.

Myers SA, Andres KR, Hagg T and Whittemore SR (2014). CD36 deletion improves recovery from spinal cord injury. Exp. Neurol. 256: 25-38.

Ni H, Jin W, Yuan B, Zhu T, et al. (2014). Curcumin inhibits the increase of labile zinc and the expression of inflammatory cytokines after traumatic spinal cord injury in rats. J. Surg. Res. 187: 646-652.

Rabchevsky AG, Patel SP and Springer JE (2011). Pharmacological interventions for spinal cord injury: where do we stand? How might we step forward? Pharmacol. Ther. 132: 15-29.

Reglodi D, Kiss P, Szabadfi K, Atlasz T, et al. (2012). PACAP is an endogenous protective factor-insights from PACAPdeficient mice. J. Mol. Neurosci. 48: 482-492.

Schonberg DL, Goldstein EZ, Sahinkaya FR, Wei P, et al. (2012). Ferritin stimulates oligodendrocyte genesis in the adult spinal cord and can be transferred from macrophages to NG2 cells in vivo. J. Neurosci. 32: 5374-5384.

Song Y, Liu J, Zhang F, Zhang J, et al. (2013). Antioxidant effect of quercetin against acute spinal cord injury in rats and its correlation with the p38MAPK/iNOS signaling pathway. Life Sci. 92: 1215-1221.

Wanner IB, Anderson MA, Song B, Levine J, et al. (2013). Glial scar borders are formed by newly proliferated, elongated astrocytes that interact to corral inflammatory and fibrotic cells via STAT3-dependent mechanisms after spinal cord injury. J. Neurosci. 33: 12870-12886.

Xianbao L, Hong Z, Xu Z, Chunfang Z, et al. (2013). Dexmedetomidine reduced cytokine release during postpartum bleeding-induced multiple organ dysfunction syndrome in rats. Mediators Inflamm. 2013: 627831.

Zeng X, Zeng YS, Ma YH, Lu LY, et al. (2011). Bone marrow mesenchymal stem cells in a three-dimensional gelatin sponge scaffold attenuate inflammation, promote angiogenesis, and reduce cavity formation in experimental spinal cord injury. Cell Transplant. 20: 1881-1899. 